\title{
The evolution of inefficiency in a simulated stag hunt
}

\author{
J. NEIL BEARDEN \\ University of North Carolina, Chapel Hill, North Carolina
}

\begin{abstract}
We used genetic algorithms to evolve populations of reinforcement learning (Q-learning) agents to play a repeated two-player symmetric coordination game under different risk conditions and found that evolution steered our simulated populations to the Pareto inefficient equilibrium under high-risk conditions and to the Pareto efficient equilibrium under low-risk conditions. Greater degrees of forgiveness and temporal discounting of future returns emerged in populations playing the low-risk game. Results demonstrate the utility of simulation to evolutionary psychology.
\end{abstract}

Traditional game theory assumes that players have perfect rationality, which implicitly assumes that they have infinite cognitive capacity and, as a result, will play Nash equilibria. Evolutionary game theory (Smith, 1982) does not necessarily endow its players with these same capabilities; rather it uses various selection processes to take populations of players to an equilibrium point over the course of generations. Other researchers have shown how learning at the individual level can lead to normative, equilibrium behavior (e.g., Fudenberg \& Levine, 1997) and can also account for the behavior of actual human players, who often exhibit nonequilibrium behavior (e.g., Atkinson, Bower, \& Crothers, 1965; Erev \& Roth, 1998; Roth \& Erev, 1995). Techniques from evolutionary computation and machine learning were used in the present study to simultaneously simulate learning at the individual level and the evolution of learning characteristics at the population level. We show that competitive forces within a population, as well as the structure of the game, can shape the behavior that emerges in an evolving population. The methodology developed here may be extended to study the evolution of behavior in other domains.

\section{The Stag Hunt Game}

Because of its interesting formal structure, the stag hunt game provides an excellent paradigm for the study of learning and evolution and how they are shaped by risk. This game, which derives its name from an oft-quoted passage from Rousseau's A Discourse on Inequality (1755), can be described as follows: Two players must coordinate their behavior in order to catch a deer. If both cooperate, each will receive enough food to last several days. If one defects on the other during the hunt and follows after a much smaller and easier to catch rabbit, the defector will

I thank Jason Arndt, Amanda Brown, Thomas S. Wallsten, Jonathan Vaughan, and two anonymous reviewers for their comments on an earlier draft of this paper. Their input improved the paper dramatically. Matlab.m files for simple genetic algorithms are available from the author by request. Correspondence regarding this article should be addressed to J. N. Bearden, Department of Psychology, The University of North Carolina, Chapel Hill, NC 27514 (e-mail: neil@unc.edu). have enough food for one day and the other will go hungry. If both follow the rabbit, each will get only a small amount of food.

Clearly, it is in the players' best interests to coordinate their actions and wait for the deer; however, a player is guaranteed food by following the rabbit. Thus, the players have a dilemma: Each must choose between potential efficiency and security. We are interested in the evolution of reinforcement learning agents that play repeated stag hunt games with one another. ${ }^{1}$ Our questions are: Will the populations evolve to choose the efficient outcome or will they evolve to choose the secure outcome? What behavioral characteristics will emerge in the evolving populations and how will these be shaped by the characteristics of the game? We will explore the process of evolution in two different risky environments. The environments differ in their penalties to the players for not coordinating their actions on an equilibrium. The two stag hunt payoff matrices that are used in this study are shown in Table 1 . The cell entries correspond to the utilities that obtain for the players for each possible outcome of the one-shot game. For example, in the top payoff matrix, when player 1 chooses $C$ and player 2 chooses $D$, player 1 earns .00 utiles and player 2 earns .40 utiles; and when both choose $D$, both earn .20 utiles, and so on. The difference between the matrices is explained below.

\section{Game Analysis}

The payoff matrices shown in Table 1 each have two pure strategy equilibria, $C C$ and $D D$. Each also has a mixed strategy equilibrium in which both players play $C$ with probability .80 . However, the latter strategy is unstable, and hence not very important; thus, we focus on the two pure strategy equilibria in this paper. ${ }^{2,3}$ In both games, $C C$ yields payoffs higher than $D D$ and is the efficient ( payoff dominant) equilibrium outcome. $D D$ is said to be the risk dominant (and inefficient) equilibrium point. ${ }^{4}$ Intuitively we can understand risk dominance by observing that $D$ offers a higher minimum payoff than $C$; that is, the worst case outcome is better when one selects $D$ than when one selects $C$, regardless of what the other player does. The bottom line is that $C C$ offers the players the 
Table 1

High- and Low-Risk Stag Hunt Matrices

High-Risk Stag Hunt $(H)$

\begin{tabular}{llll} 
& & \multicolumn{2}{c}{ Player 2 } \\
\cline { 3 - 3 } Player 1 & & $C$ & $\frac{D}{C}$ \\
\cline { 3 - 4 } & $D$ & $.45, .45$ & $.00, .40$ \\
& $D$ & $.40, .00$ & $.20, .20$
\end{tabular}

Low-Risk Stag Hunt $(L)$

\begin{tabular}{cc}
\multicolumn{2}{c}{ Player 2 } \\
\hline$\frac{C}{.45, .45}$ & $\frac{D}{.00, .43}$ \\
$.43, .00$ & $.08, .08$
\end{tabular}

Note-Matrix elements represent von Newmann and Morgenstern utilities.

best outcome, collectively and individually, but choosing $D$ guarantees a player a higher minimum payoff.

The payoff matrices differ in the penalties that obtain for failing to coordinate on an equilibrium. The penalties in the payoff matrix shown in the top of Table 1 are greater than the corresponding deviations in the matrix in the bottom. (Throughout, we will refer to these matrices as $H$ and $L$, respectively.) In $H$, a player loses (or fails to gain) . 20 for choosing $C$ when its opponent chooses $D$ and loses .05 for choosing $D$ when its opponent chooses $C$. The corresponding losses are .08 and .02 in $L$. Thus, the losses are 2.5 times greater in $H$ than in $L$. The former will be referred to as the high-risk stag hunt game and the latter as the low-risk stag hunt game.

Again, although both of our games have the same equilibria and the same best reply structure, they differ in their levels of risk: Making the wrong choice in $H$-that is, deviating from equilibrium when one's opponent stays in equilibrium - is more costly (risky) than the corresponding wrong choice in $L$. Battalio, Samuelson, and van Huyck (2000) referred to this penalty as an optimization premium and found that higher optimization premiums were more likely to lead human players to the risk dominant equilibrium in a laboratory stag hunt game. In their experiment, on each trial, individuals in a cohort were randomly paired with one another and played one round of the stag hunt game. Each of the cohorts played one of three different stag hunt matrices that had the same pure and mixed equilibria but that differed in their optimization premiums. On the first trial, there was no clear preference for either $C C$ or $D D$ across the three matrices; however, the groups with the higher optimization premiums (i.e., with greater risk) became progressively less likely to play $C C$ throughout the game. They learned to play the Pareto inefficient equilibrium. In the present study, we did not use a game of this sort. Our simulated players were randomly paired and played repeated games (rather than single-shot games) with one another. Despite this difference, our results are (ostensibly) very similar to those obtained by Battalio et al.

In games with multiple equilibria, such as those studied here, traditional game theory does not prescribe which of the available equilibria the players ought to choose, only that they choose one. In their influential work on the equilibrium selection problem, Harsanyi and Selten (1988) gave priority to the payoff dominant equilibrium, but other theorists have argued for the risk dominant equilibrium (e.g., Carlsson \& van Damme, 1993). Given this disagreement, it is not intuitively clear how we should expect nature to solve this problem. Evolution is often thought of as an optimization process; so, assuming this Panglossian notion of evolution, we might expect evolving species of players to tend toward efficient equilibria, which is, presumably, the best possible outcome for both the individual player and the population. 5 As will be shown below, the results of (simulated) natural selection are not always consistent with a priori intuitions of optimality.

\section{Autonomous Agents and Q-Learning}

Our species of autonomous agent is the Q-learning algorithm. The Q-learning algorithm learns reinforcements associated with state-action pairs and adjusts its behavior over time in order to maximize its reinforcement (Watkins, 1989). Here, the algorithms play one another in repeated stag hunt games, with each trying to maximize its own earnings. There are several models of reinforcement learning in the psychological literature that have been applied to learning in simple games (e.g., Atkinson et al., 1965; Bush \& Mosteller, 1955; Erev \& Roth, 1998; Sandholm \& Crites, 1996). We use Q-learning because of its remarkable success in other interesting applications. For example, Tesauro (1995) showed that a neural network based on Q-learning principles is capable of learning to play backgammon as well as master human players do. Another virtue of the Q-learning algorithm is that its parameters have clear, meaningful psychological interpretations, and its parameters are few. Together, these make the Q-learning algorithm an ideal candidate for a model of human learning in game theoretic situations. (See Fudenberg \& Levine, 1997, for a rigorous overview of learning models in game theory.)

Sandholm and Crites (1996) showed that Q-learning agents can learn to play optimally against tit-for-tat in the prisoner's dilemma but have a difficult time learning to cooperate among themselves. These authors assumed particular parameter values for the algorithms in their experiments. We take a different approach and use genetic search to find optimal (or quasi-optimal) parameters for our stag hunting agents. This allows us to look at the behavioral characteristics that evolve in different risky environments. Rather than arbitrarily setting the parameters and looking at their consequences, we introduce a computational procedure for finding parameter values. The procedure is useful both as a general optimization method and as a means of examining the dynamics of evolving populations.

In order to make their decisions, our Q-learning agents estimated the expected future discounted rewards (Qvalues) for each available action $(C$ and $D)$ in each possible state $(C C, D D, C D$, and $D C)$, where a state is defined as the outcome of the previous game. For example, the agents estimate the reward they expect to receive for 
choosing $C$ when in the state $C D$ and then for taking the action with the highest Q-value in each subsequent state. The reinforcements expected to be received in subsequent states are time discounted to capture the psychological characteristic that the same reinforcement is believed to be worth more now than it is expected to be worth later (e.g., Thaler \& Shefrin, 1981). As the agents play the game, they update their Q-values with a method similar to dynamic programming.

The basic procedure for updating Q-values can be described as follows: After taking an action $a$ while in a state $s$, receiving reward $r$, and moving into a new state $s^{\prime}$, the agents refine their estimate of their expected future discounted rewards for taking action $a$ in state $s$ and then taking the optimal action in each subsequent state (i.e., taking the action with the highest Q-value), denoted $\mathrm{Q}(a, s)$ with the following expression:

$$
Q(a, s) \leftarrow(1-\alpha) Q(a, s)+\alpha\left(r+\gamma \max _{a^{\prime}} Q\left(a^{\prime}, s^{\prime}\right)\right),
$$

where $0<\alpha, \gamma<1$. The learning parameter $\alpha$ governs the rate at which Q-values are updated, and the discount parameter $\gamma$ determines how much the agent currently values the reinforcement it expects to receive in the future. As $\gamma$ approaches 1 , the agent values future reinforcement as much as it values current reinforcement; as $\gamma$ approaches 0 , the agent cares only about its current reinforcement and does not consider future returns. Q-learning agents attempt to learn the correct Q-values for the environment in which they operate, so it is important that they explore their environment before settling on a particular policy. If an agent always selected the action in a particular state that had the highest (current) Q-value, it might fail to take another available action that would lead to a higher return. Only through exploring the available actions can an agent learn the correct Q-values associated with the actions. Our agents use an $\varepsilon$-greedy approach to solve the exploration problem (Sutton \& Barto, 1998). On each trial the agent randomly selects an available action (explores) with probability $\varepsilon$ and, with $1-\varepsilon$, selects the available action with the highest Q-value (exploits). We take an annealing approach and decrease $\varepsilon$ each iteration of the repeated game. The probability of exploration decreases exponentially as the repeated game continues until it reaches $\varepsilon_{\min }$ on the final trial of the game. This allows the agent to explore its available actions and to exploit the best available action more often as it gets better estimates of the Q-values associated with its actions. The parameter $\varepsilon_{\min }$ will be referred to as exploration rate in what follows.

All agents use Equation 1 to update their Q-values and differ only in their parameter settings. In game theoretic terms, each agent's strategy can be completely represented by Equation 1 and by its learning rate $\alpha$, discount factor $\gamma$, and exploration rate $\varepsilon_{\min }$. Q-learning requires that the agents have only minimal computational resources. The agents represent the playing space with only four states and have only two possible actions $(C$ and $D)$ available in each state. Thus, they need only keep track of eight
Q-values. Hence our learning agents are, at best, boundedly rational (Simon, 1957).

\section{Genetic Algorithms}

Genetic algorithms (Holland, 1975) have been used to model sexual selection (Collins \& Jefferson, 1992), ecosystems (Holland, 1995), artificial stock markets (Palmer, Arthur, Holland, LeBaron, \& Tayler, 1994), and many other complex systems. As one highly relevant example, Axelrod (1987) used genetic algorithms (GAs) to evolve strategies for the iterated prisoner's dilemma game (IPD) and found strategies that could outperform tit-for-tat, which is the most oft-cited and one of the most robust strategies for the IPD, in an ecology that was made up of eight different strategies created by game theorists. In the present paper, GAs are used both to model the evolution of a system of interacting agents in the stag hunt game and to find optimal (or quasi-optimal) parameter values for the Q-learning algorithm for our different payoff matrices. (For an introduction to and a broad overview of genetic algorithms, see Goldberg, 1989, and Mitchell, 1996.)

Each simulated agent is represented by a binary string. The string encodes the agent's two basic Q-learning parameter values and the value of its exploration rate, with each parameter encoded in 10 bits of a 30 bit string. This coding method allows us to conveniently represent realvalued parameters as binary strings and, most importantly, improves the performance of the GA (Goldberg, 1989).

For each stag hunt matrix, we conducted 64 runs of 50 generations of a simple genetic algorithm (Mitchell, 1996). Each agent in each initial population was created by assigning each bit of its chromosome a " 1 " or " 0 " with equal probability. Each agent in each generation played six other randomly chosen agents from the population in stag hunt games of 1,000 iterations each. The GA parameters we used were chosen on the basis of suggestions of other researchers who have used GAs to optimize functions (e.g., De Jong, 1975) and our own experimentation.

The genetic algorithm is a simple way to mimic natural selection. The agents that do well in the stag hunt gamethat is, those who earn a lot-are more likely to reproduce and pass their genetic material on to subsequent generations. The mating procedure combines the genetic material of different agents, resulting in combinations of parameter values that were perhaps not present in the initial generation, and mutation maintains a flow of new, perhaps better, genes into the population. It is important to state that the simulations are not Lamarckian in any way. The agents' genes (i.e., their parameters) and not what they learn are passed on to subsequent generations.

\section{Results}

Equilibrium selection. The simulations produced several consistent results. First, the populations of Q-learning agents evolved to choose the payoff-dominant equilibrium $(C C)$ more often in the low-risk (low optimization premium) stag hunt $(L)$ than in the high-risk stag hunt $(H)$. See Figure 1. By the final generation, agents in the 


\section{High Risk}

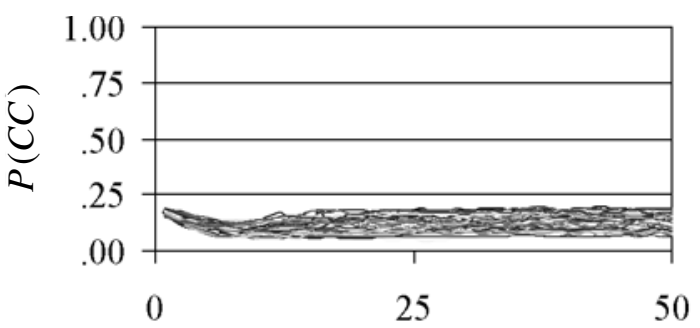

Generation

\section{Low Risk}

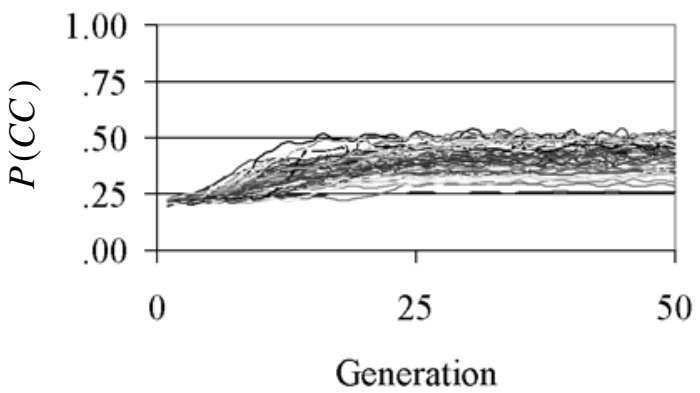

Figure 1. Proportion of $C C$ plays in each generation for each run for the $H$ and $L$ stag hunt matrices.

$L$ game selected the payoff-dominant equilibrium almost $50 \%$ of the time, whereas the agents in the $H$ game selected it only about $10 \%$ of the time. Secondly, the populations evolved to choose the risk dominant equilibrium (DD) almost $75 \%$ of the time in the $H$ stag hunt and typically less than $35 \%$ of the time in the $L$ stag hunt. ${ }^{6}$ See Figure 2. And the rate at which the agents converged to their respective prominent equilibrium was greater for $H$ than for $L$.

Earnings. In the final generations, the agents in the $H$ game earned about .23 utiles per interaction, and the agents in the $L$ matrix earned about .28 utiles per interaction. Interestingly, if the agents had played randomly, the populations playing the $H$ game would have earned more than they did (expected value $=.26$ utiles/interaction), whereas agents in the $L$ game would have earned less than they did (expected value $=.24$ utiles/interaction). This highlights how surprising the equilibrium selection results are: The agents in $H$ evolved to earn less than completely random agents. Earnings in the high-risk $(H)$ games consistently dropped in the early generations and then increased to their steady state by about the 15 th generation. In the early generations, many of the agents, owing to their high exploration rates, randomly took their actions on a large number of trials. The payoffs decreased in the early generations in $H$ as the average exploration rate decreased and the agents failed to synchronize their behaviors; it then increased as the agents (stochastically) locked into equilibrium. This initial drop-off in earnings followed by an increase has been found in other game theoretic simulations that start with a random population, though for different reasons (see, e.g., Axelrod, 1987).

Exploration rates. For both payoff matrices, the populations converged to exploration rates $\left(\varepsilon_{\min }\right)$ of less than .05 by about the 10 th generation. The rate at which the average population exploration rate decreased was greater for $H$ than for $L$ due to the greater penalties for nonequilibrium play in $H$. The exploration rate was the most powerful determinant in the early course of the evolution of agents in both matrices. Once the exploration rate reached a steady state, the learning and discount rates started to shape the course of evolution.

Learning rates. The learning parameter $(\alpha)$ tended toward values below .30 for the $L$ but did not converge for the $H$ matrix. We found that high learning rates in the $L$ matrix prevented the agents from maintaining the efficient equilibrium as easily as agents with lower learning rates. The high learning rate agents ceased to cooperate with only slight provocation, whereas the low learning rate agents were less easily provoked and were more likely to maintain the efficient equilibrium. In the $H$ matrix, nearly all learning rates were successful, or, rather, the populations did not converge to a particular learning rate in the $H$ matrix. The learning parameter seems to cap-

High Risk

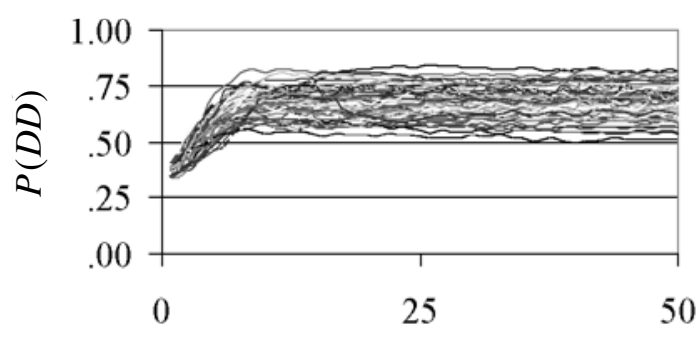

Generation

Low Risk

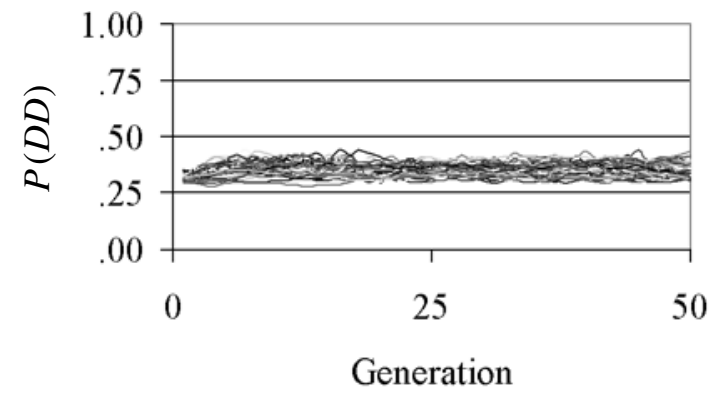

Figure 2. Proportion of $D D$ plays in each generation for each run for the $H$ and $L$ stag hunt matrices. 
ture forgiveness (or reactance) in the learning agents, with lower learning rates corresponding to higher levels of forgiveness. Axelrod (1984) and Godfray (1992) used results from prisoner's dilemma simulations to argue that natural selection favors individuals who are forgiving. However, we see from our results that the survival advantage of forgiveness depends on characteristics of the environment (or game) and is not necessarily a universally important survival characteristic.

Discount rates. The discount parameter $(\gamma)$, which determines how much individuals currently value future returns, tended toward higher values (i.e., lower discounting) for the $H$ matrix (around $\gamma=.75$ ) than for the $L$ matrix (around $\gamma=.25$ ). This finding is consistent with experimental data that show that people discount outcomes with smaller absolute magnitudes more than they do outcomes with larger absolute magnitudes (Loewenstein \& Prelec, 1989; Prelec \& Loewenstein, 1991). There is a debate as to whether or not temporal discounting is rational (see, e.g., Schick, 1997). Our results suggest, perhaps, that it is and that the magnitude of discounting should be governed by the environment.

\section{DISCUSSION}

The populations showed consistent evolutionary patterns, nearly always tending toward the efficient (payoff dominant) equilibrium when the penalty for not choosing a best response was small and toward the safe (risk dominant) equilibrium when the penalty was large. The pattern of results in the final generations is consistent with behavioral data from human participants (Battalio et al., 2000). These results raise questions about the soundness of the intuitive argument that efficiency is the rational outcome of the stag hunt game. We cannot safely draw that conclusion without considering the risk associated with the hunt. Our species of stag hunters evolved in stochastic, risky environments, and natural selection did not always favor individuals playing the payoff dominant alternative. If survival depends on inefficiency in certain environments, inefficiency seems to be the rational strategy.

One of the fundamental assumptions of evolutionary psychology is that one must look at the environment in which an organism has evolved in order to understand why it behaves the way it does (Cosmides \& Tooby, 1987). We found that our two different games led to systematically different evolutionary outcomes. For example, in the highrisk game we found that the agents typically evolved to be less forgiving than the agents who evolved in the lowrisk game, because in the former it was more costly to be "betrayed" than in the latter. Since coordination was more important in the high-risk game and lower levels of forgiveness emerged, we might expect cultures in which interdependence is high to show less forgiveness than those in which it is low. This consequence seems counterintuitive and highlights the utility of simulation in generating testable predictions. One could easily extend the procedures developed in this paper to simulate other types of environments and to look at the behavioral characteristics that evolve in those environments. These computational procedures can provide a firm methodological framework within which to both generate and test hypotheses regarding adaptation and human behavior.

\section{REFERENCES}

Atkinson, R. C., Bower, G. H., \& Crothers, E. J. (1965). An introduction to mathematical learning theory. New York: Wiley.

AXElrod, R. (1984). The evolution of cooperation. New York: Basic Books.

AxELrod, R. (1987). The evolution of strategies in the iterated prisoner's dilemma. In L. Davis (Ed.), Genetic algorithms and simulated annealing (pp. 32-41). Los Altos, CA: Morgan Kaufman.

Battalio, R., Samuelson, L., \& van Huyck, J. (2000). Optimization incentives and coordination failure in laboratory stag hunt games. Unpublished manuscript.

BuSH, R, \& Mosteller,F. (1955). Stochastic models of learning. New York: Wiley.

CARlsson, E., \& van DAMme, E. (1993). Global games and equilibrium selection. Econometrica, 61, 989-1018.

Collins, R. J., \& Jefferson, D. R. (1992). The evolution of sexual selection and female choice. In F. J. Valera \& P. Bourgine (Eds.), Towards a practice of autonomous systems: Proceedings of the first European conference on artificial life (pp. 327-336). Cambridge, MA: MIT Press.

Cosmides, L., \& Tоову, J. (1987). From evolution to behavior: Evolutionary psychology as the missing link. In J. Dupre (Ed.), The latest on the best: Essays on evolution and optimality (pp. 276-306). Cambridge, MA: MIT Press.

DAwKIns, R. (1976). The selfish gene. Oxford: Oxford University Press. DE JONG, K. A. (1975). An analysis of the behavior of a class of genetic adaptive systems. Unpublished doctoral dissertation, University of Michigan.

Erev, I., \& Roth, A. E. (1998). Predicting how people play games: Reinforcement learning in experimental games with unique, mixed strategy equilibria. American Economic Review, 88, 848-881.

FudenberG, D., \& LeVine, D. K. (1997). The theory of learning in games. Cambridge, MA: MIT Press.

Fudenberg, D., \& Tirole, J. (1991). Game theory. Cambridge, MA: MIT Press.

Godfray, H. C. (1992). The evolution of forgiveness. Nature, $\mathbf{3 5 5}$, 206-207.

GoldBERG, D. E. (1989). Genetic algorithms: In search, optimization, and machine learning. Reading, MA: Addison-Wesley.

Harsanyi, J. C., \& Selten, R. (1988). A general theory of equilibrium selection in games. Cambridge, MA: MIT Press.

Holland, J. H. (1975). Adaptation in natural and artificial systems. Ann Arbor: University of Michigan Press.

Holland, J. H. (1995). Hidden order. Reading, MA: Helix Books.

Loewenstein, G. F., \& Prelec, D. (1989). Anomalies in intertemporal choice: Evidence and an interpretation. Unpublished working paper. Chicago: University of Chicago, Graduate School of Business, Center for Decision Research.

Mitchell, M. (1996). An introduction to genetic algorithms. Cambridge, MA: MIT Press.

Palmer, R. G., Arthur, W. B., Holland, J. H., LeBaron, B., \& TayLER, P. J. (1994). Artificial economic life: A simple model of a stock market. Physica D, 75, 264-274.

Prelec, D., \& Loewenstein, G. F. (1991). Decision making over time and under uncertainty: A common approach. Management Science, 37, 770-786.

Roth, A. E., \& Erev, I. (1995). Learning in extensive-form games: Experimental data and simple dynamic models in the intermediate term. Games \& Economic Behavior, 8, 164-212.

Rousseau, J. J. (1984). A discourse on inequality (M. Cranston, Trans.). London: Penguin. (Original work published 1755)

Sandholm, T. W., \& Crites, R. H. (1996). Multiagent reinforcement learning in the iterated prisoner's dilemma. Biosystems, 37, 148-166. ScHICK, F. (1997). Making choices: A recasting of decision theory. New York: Cambridge University Press. 
Simon, H. A. (1957). Models of man. New York: Wiley.

Sмгтн, J. M. (1982). Evolution and the theory of games. Cambridge: Cambridge University Press.

Sutton, R. S., \& BARTo, A. G. (1998). Reinforcement learning: An introduction (adaptive computation and machine learning). Cambridge, MA: MIT Press.

Tesauro, G. (1995). Temporal difference learning and TD-Gammon. Communications of the ACM, 38, 58-68.

Thaler, R., \& Shefrin, H. (1981). An economic theory of self-control. Journal of Political Economy, 89, 392-406.

WAT KINS, C. (1989). Learning form delayed rewards. Unpublished doctoral dissertation, University of Cambridge.

WeIBUll, J. W. (1995). Evolutionary game theory. Cambridge, MA: MIT Press.

\section{NOTES}

1. Following the tradition in distributed artificial intelligence, we will (sometimes) refer to our simulated players as "agents."

2 . The payoff for $D C$ need not exceed the payoff for $D D$ for the game to be a stag hunt. Some sources also refer to games in which $C C>D C=$ $D D>C D$ as stag hunts (see Fudenberg \& Tirole, 1991).

3. Technically, we explore repeated play of the stag game in which the number of repetitions is unknown to the agents and, as far as they know, is infinite; so, according to the folk theorem, our agents could, if they were sufficiently patient, establish other equilibria. Our agents are sufficiently simple that we feel justified in looking at their behavior in terms of the single-shot equilibria.

4. $C C$ is said to be the payoff dominant equilibrium since it is not Pareto dominated by any other equilibrium point (Harsanyi \& Selten, 1988). $D D$ is the risk dominant equilibrium because it Pareto dominates $C C$ when the payoff matrices are normalized (Weibull, 1995).

5. The forces behind evolution are the subject of interesting scientific and philosophical debates that go far beyond the scope of this paper. We will work with the simple species survival optimization notion of evolution. For an alternative (and more well-informed notion), see Dawkins (1976).

6. Essentially the same pattern of equilibrium behavior obtained when we ran separate simulations using Erev and Roth's (1998) single parameter reinforcement learning model, which is based on Bush and Mosteller's (1955) model of stochastic learning. Thus, our findings do not seem to be an artifact of the peculiarities of our learning mechanism; rather, they seem to be a general consequence of stochastic reinforcement learning.

(Manuscript received November 16, 2000; revision accepted for publication March 11, 2001.)= 